\title{
PERTURBED FREDHOLM BOUNDARY VALUE PROBLEMS FOR DELAY DIFFERENTIAL SYSTEMS
}

\author{
ALEXANDER A. BOICHUK \\ AND MYRON K. GRAMMATIKOPOULOS
}

Received 3 March 2003

Boundary value problems for systems of ordinary differential equations with a small parameter $\varepsilon$ and with a finite number of measurable delays of the argument are considered. Under the assumption that the number $m$ of boundary conditions does not exceed the dimension $n$ of the differential system, it is proved that the point $\varepsilon=0$ generates $\rho$-parametric families (where $\rho=n-m$ ) of solutions of the initial problem. Bifurcation conditions of such solutions are established. Also, it is shown that the index of the operator, which is determined by the initial boundary value problem, is equal to $\rho$ and coincides with the index of the unperturbed problem. Finally, an algorithm for construction of solutions (in the form of Laurent series with a finite number terms of negative power of $\varepsilon$ ) of the boundary value problem under consideration is suggested.

\section{Introduction}

We consider in Banach spaces the problem of existence and construction of solutions $z:[a, b] \rightarrow \mathbb{R}^{n}$ of systems of ordinary differential equations with a small parameter $\varepsilon$ and with a finite number of measurable delays of argument of the form

$$
\dot{z}(t)=\sum_{i=1}^{k} A_{i}(t) z\left(h_{i}(t)\right)+\varepsilon \sum_{i=1}^{k} B_{i}(t) z\left(h_{i}(t)\right)+g(t), \quad t \in[a, b], h_{i}(t) \leq t,
$$

with the initial conditions

$$
z(s)=\psi(s), \quad \text { if } s<a<b,
$$

and subject to the boundary conditions

$$
l z=\alpha, \quad \alpha \in \mathbb{R}^{m} .
$$

Copyright (C) 2003 Hindawi Publishing Corporation Abstract and Applied Analysis 2003:15 (2003) 843-864 2000 Mathematics Subject Classification: 34K10, 34K06, 34K18 URL: http://dx.doi.org/10.1155/S1085337503304026 
In this connection, we suppose that the unperturbed problem $(\varepsilon=0)$ does not have solutions for arbitrary nonhomogeneities $g(t)$ belonging to the space considered below and $\alpha \in \mathbb{R}^{m}$ and for arbitrary initial function $\psi: \mathbb{R}^{1} \backslash[a, b] \rightarrow$ $\mathbb{R}^{n}$. Moreover, we suppose that the number $m$ of boundary conditions (1.3) does not exceed the dimension $n$ of the differential system (1.1). Further, we establish conditions for the perturbed coefficients $B_{i}(t)$ and for the delays $h_{i}(t)$, under which the boundary value problem (1.1) and (1.3) admits a family of solutions or a single solution. Finally, we suggest an algorithm for the construction of such solutions.

In the case where there is no delay effect $\left(h_{i}(t)=t, i=1, \ldots, k\right)$ and $m=n$, problem (1.1) and (1.3) has been studied in [2, page 252]. Also, in the case where there is no delay effect $\left(h_{i}(t)=t, i=1, \ldots, k\right)$ and $A_{i}(t)=0$, the periodic $(l z:=$ $z(a)-z(b)=0$ ) boundary value problem (1.1) and (1.3) has been considered in [6].

\section{Initial value problems}

Consider the linear equation with concentrated delay

$$
\begin{gathered}
\dot{z}(t)-\sum_{i=1}^{k} A_{i}(t) z\left(h_{i}(t)\right)=g(t), \quad t \in[a, b], \\
z(s)=\psi(s), \quad \text { if } s<a,
\end{gathered}
$$

where $A_{i}(t)$ are $n \times n$ matrices, while the functions $h_{i}(t) \leq t$ are measurable for $t \in[a, b]$.

Usually (see $[3,8]$ ), a solution of the delay differential equation (2.1) is constructed in the space of continuously differentiable functions as a continuous extension of the initial function $\psi(s)$ to the interval $[a, b]$. Such a definition requires the initial function $\psi(s)$ and the solution $z(s)$ to be "continuously joined" at the point $s=a$, that is, $\psi(a)=z(a)$. This leads to the notion of infinitedimensional fundamental matrix (introduced for the investigation of the initial problem (2.1)) whose dimension coincides with the dimension of the basis of the space of initial functions.

Following [1], we will present here basic notions concerning the initial problem (2.1) for delay differential systems with a finite-dimensional fundamental matrix.

Let $h_{i}:[a, b] \rightarrow \mathbb{R}^{1}$ and $\psi: \mathbb{R}^{1} \backslash[a, b] \rightarrow \mathbb{R}^{n}$ be given functions. Define

$$
\left(S_{h_{i}} z\right)(t)= \begin{cases}z\left(h_{i}(t)\right), & \text { if } h_{i}(t) \in[a, b], \\ 0, & \text { if } h_{i}(t) \notin[a, b],\end{cases}
$$

where $S_{h_{i}}$ denotes (see [1, page 10]) the operator of so-called inner composition, and put 


$$
\psi^{h_{i}}(t)= \begin{cases}0, & \text { if } h_{i}(t) \in[a, b], \\ \psi\left(h_{i}(t)\right), & \text { if } h_{i}(t) \notin[a, b] .\end{cases}
$$

Now, in view of (2.2) and (2.3), (2.1) can be rewritten in the form

$$
(L z)(t):=\dot{z}(t)-\sum_{i=1}^{k} A_{i}(t)\left(S_{h_{i}} z\right)(t)=\varphi(t),
$$

where

$$
\varphi(t)=g(t)+\sum_{i=1}^{k} A_{i}(t) \psi^{h_{i}}(t)
$$

The transformations (2.2) and (2.3) allow to join the initial function $\psi(s)$, $s<a$, of (2.1) and the absolute term and to apply to (2.4) the well-developed methods of linear functional analysis. We will investigate (2.4) under the assumption that the operator $L$ bounded on $[a, b]$ maps the Banach space $D_{p}^{n}[a, b]$ of absolutely continuous functions $z:[a, b] \rightarrow \mathbb{R}^{n}$ with the norm

$$
\|z\|_{D_{p}^{n}}=\|\dot{z}\|_{L_{p}^{n}}+\|z(a)\|_{\mathbb{R}^{n}}
$$

into the Banach space $L_{p}^{n}[a, b](1<p<\infty)$ of integrable vector functions $\varphi$ : $[a, b] \rightarrow \mathbb{R}^{n}$ with the norms standard in these spaces.

According to [1, page 13], the vector function $z(t) \in D_{p}^{n}[a, b]$, for which $\dot{z}(t) \in$ $L_{p}^{n}[a, b]$ and which is absolutely continuous on $[a, b]$, is called a solution of the delay differential system (2.4) if $z(t)$ satisfies the system (2.4) almost everywhere on $[a, b]$.

In the sequel, we will consider (2.4) rewritten in the form

$$
\dot{z}(t)=A(t)\left(S_{h} z\right)(t)+\varphi(t) .
$$

Here $A(t)=\left(A_{1}(t), \ldots, A_{k}(t)\right)$ is an $n \times N$ matrix $(N=n k)$ consisting of $n \times n$ matrices $A_{i}(t),\left(S_{h} z\right)(t)=\operatorname{col}\left[\left(S_{h_{1}} z\right)(t), \ldots,\left(S_{h_{k}} z\right)(t)\right]$ is an $N$-dimensional column vector, and $\varphi(t)$ is an $n$-dimensional column vector given by (2.5). The operator of inner composition $S_{h}$ maps the space $D_{p}^{n}$ into the space

$$
L_{p}^{N}=\underbrace{L_{p}^{n} \times \cdots \times L_{p}^{n}}_{k \text { time }}
$$


that is, $S_{h}: D_{p}^{n} \rightarrow L_{p}^{N}$. For the operator $S_{h_{i}}: D_{p}^{n} \rightarrow L_{p}^{n}$, we have the following representation:

$$
\left(S_{h_{i}} z\right)(t)=\int_{a}^{b} \chi_{h_{i}}(t, s) \dot{z}(s) d s+\chi_{h_{i}}(t, a) z(a),
$$

where $\chi_{h_{i}}(t, s)$ is the characteristic function of the set

$$
\Omega=\left\{(t, s) \in[a, b] \times[a, b]: a \leq s \leq h_{i}(t) \leq b\right\}
$$

and it means (see [1, page 17] or [4]) that

$$
\chi_{h_{i}}(t, s)= \begin{cases}1, & \text { if }(t, s) \in \Omega, \\ 0, & \text { if }(t, s) \notin \Omega .\end{cases}
$$

It is well known that the nonhomogeneous delay operator equation (2.4) is solvable for any right-hand side $\varphi(t) \in L_{p}^{n}[a, b]$ and admits an $n$-parametric family of solutions in the form

$$
z(t)=X(t) c+\int_{a}^{b} K(t, \tau) \varphi(\tau) d \tau,
$$

where the $n \times n$ matrix $K(t, \tau)$ is called Cauchy matrix. For any fixed $\tau$, this matrix is a solution to the following matrix Cauchy problem:

$$
\frac{\partial K(t, \tau)}{\partial t}=A(t)\left(S_{h} K(\cdot, \tau)\right)(t), \quad K(\tau, \tau)=I .
$$

In what follows, we assume that the matrix $K(t, \tau)$ is defined in the square $[a, b] \times$ $[a, b]$, where $K(t, \tau) \equiv 0$ for $a \leq t<\tau \leq b$. The finite-dimensional fundamental $n \times n$ matrix of the homogeneous $(\varphi(t) \equiv 0)$ delay equation corresponding to (2.4) is of the form $X(t)=K(t, a)$. By $\left(S_{h} K(\cdot, \tau)\right)(t)$ we denote the $N \times n$ matrix whose columns are obtained by applying the operator of inner composition $S_{h}$ to the corresponding columns of $n \times n$ matrix $K(t, \tau)$.

\section{Fredholm boundary value problems}

Consider the following linear nonhomogeneous boundary value problem:

$$
\begin{gathered}
(L z)(t):=\dot{z}(t)-A(t)\left(S_{h} z\right)(t)=\varphi(t), \quad t \in[a, b], \\
l z=\alpha .
\end{gathered}
$$

Here $L: D_{p}^{n}[a, b] \rightarrow L_{p}^{n}[a, b]$ is the bounded linear delay differential operator, $l=\operatorname{col}\left[l_{1}, \ldots, l_{m}\right]$ is an $m$-dimensional bounded vector functional, the number $m$ of components which, in general, is not equal to the dimension $n$ of the differential system. Functionals $l_{i}$ map the space $D_{p}^{n}[a, b]$ into the space $\mathbb{R}$, while $l: D_{p}^{n}[a, b] \rightarrow \mathbb{R}^{m} ; \alpha \in \mathbb{R}^{m}$. Moreover, the rows of the matrices $A_{i}(t)$ and the column vector $\varphi(t)$ belong to the space $L_{p}^{n}[a, b]$, that is, $A_{i}(t), \varphi(t) \in L_{p}^{n}[a, b]$. It is 
well known (see [1, page 33] or [7, page 86]) that this boundary value problem defines a Fredholm operator, which maps the space $D_{p}^{n}[a, b]$ into the space $L_{p}^{n}[a, b] \times \mathbb{R}^{m}$.

Here we are interested in necessary and sufficient conditions for solvability of the above problem as well as in finding a representation of its solution $z(t) \in$ $D_{p}^{n}[a, b]$.

The general solution of (3.1) is of the form (2.12). So, substituting (2.12) into the boundary conditions (3.2), we obtain the algebraic (with respect to $c \in \mathbb{R}^{n}$ ) system

$$
Q c=\alpha-l \int_{a}^{b} K(\cdot, \tau) \varphi(\tau) d \tau
$$

with the $(m \times n)$-dimensional constant matrix $Q=l X(\cdot)$ and with $\operatorname{rank} Q=n_{1}$. From the system (3.3) we can find the constant $c \in \mathbb{R}^{n}$ for which the solution (2.12) of the system (3.1) is also a solution of the boundary value problem (3.1) and (3.2).

Using the theory of pseudoinverse matrices and orthoprojectors (see, e.g., [9] or [2, Theorem 3.9, page 92]), we receive necessary and sufficient conditions for solvability of the algebraic system (3.3) and for the existence of solutions for the boundary value problem (3.1) and (3.2).

Let $P_{Q}: \mathbb{R}^{n} \rightarrow N(Q)=\operatorname{ker} Q$ and $P_{Q^{*}}: \mathbb{R}^{m} \rightarrow N\left(Q^{*}\right)=\operatorname{ker} Q^{*}=\operatorname{coker} Q$ denote, respectively, the $(n \times n)$ - and $(m \times m)$-dimensional matrices-orthoprojectors on the kernel and the cokernel of the matrix $Q$ with the properties $P_{Q}^{2}=$ $P_{Q}=P_{Q}^{*}, P_{Q^{*}}^{2}=P_{Q^{*}}=P_{Q^{*}}^{*}$, where the symbol $*$ means the operation of transposition. Also let $Q^{+}$denote the $n \times m$ matrix, which is a Moore-Penrose pseudoinverse to $Q$.

The algebraic system (3.3) is solvable if and only if its right-hand side belongs to the orthogonal complement $N^{\perp}\left(Q^{*}\right)=\mathbb{R}(Q)$ of the subspace $N\left(Q^{*}\right)$. This means that the equality

$$
P_{Q^{*}}\left\{\alpha-l \int_{a}^{b} K(\cdot, \tau) \varphi(\tau) d \tau\right\}=0
$$

holds. Since $\operatorname{rank} P_{Q}=n-\operatorname{rank} Q=n-n_{1}=r$ and $\operatorname{rank} P_{Q^{*}}=m-\operatorname{rank} Q^{*}=$ $m-n_{1}=d$, we use the symbol $P_{Q_{d}^{*}}$ to denote the $d \times m$ matrix whose rows represent a complete set of $d$ linearly independent rows of the $m \times m$ matrix $P_{Q^{*}}$. Let $P_{Q_{r}}$ be an $n \times r$ matrix whose columns represent a complete set of $r$ linearly independent columns of the $n \times n$ matrix $P_{Q}$. Then the last condition is expressed by the equality

$$
P_{Q_{d}^{*}}\left\{\alpha-l \int_{a}^{b} K(\cdot, \tau) \varphi(\tau) d \tau\right\}=0
$$


If (3.5) holds, then

$$
c=Q^{+}\left(\alpha-l \int_{a}^{b} K(\cdot, \tau) \varphi(\tau) d \tau\right)+P_{Q_{r}} c_{r}, \quad P_{Q_{r}} c_{r} \in N(Q), \forall c_{r} \in \mathbb{R}^{r}
$$

is a solution of the algebraic system (3.3). Substituting the obtained value of $c$ into (2.12), we receive the general solution of the boundary value problem (3.1) and (3.2)

$$
\begin{aligned}
z(t, c)= & X(t) P_{Q_{r}} c_{r}+X(t) Q^{+} \alpha+\int_{a}^{b} K(t, \tau) \varphi(\tau) d \tau \\
& -X(t) Q^{+} l \int_{a}^{b} K(\cdot, \tau) \varphi(\tau) d \tau .
\end{aligned}
$$

This solution can be rewritten in the form

$$
z\left(t, c_{r}\right)=X_{r}(t) c_{r}+(G \varphi)(t)+X(t) Q^{+} \alpha
$$

where $X_{r}(t)=X(t) P_{Q_{r}}$ is the fundamental matrix of the homogeneous boundary value problem

$$
\dot{z}(t)=A(t)\left(S_{h} z\right)(t), \quad l z=0 .
$$

The operator $(G \varphi)(t)$ is defined as

$$
(G \varphi)(t)=\int_{a}^{b} K(t, \tau) \varphi(\tau) d \tau-X(t) Q^{+} l \int_{a}^{b} K(\cdot, \tau) \varphi(\tau) d \tau
$$

and is called generalized Green operator for the boundary value problem (3.1) and (3.2) (see [2, page 134]).

From the above observation follows the following theorem.

Theorem 3.1. Consider the boundary value problem (3.1) and (3.2). Then

(1) the operator $\Lambda_{0}: D_{p}^{n}[a, b] \rightarrow L_{p}^{n}[a, b] \times \mathbb{R}^{m}$ defined by the formula

$$
\left(\Lambda_{0} z\right)(t) \stackrel{\text { def }}{=} \operatorname{col}\left[\dot{z}(t)-A(t)\left(S_{h} z\right)(t), l z\right]
$$

is a Fredholm one with

$$
\text { ind } \Lambda_{0}=\operatorname{dim} \operatorname{ker} \Lambda_{0}-\operatorname{dim} \operatorname{ker} \Lambda_{0}^{*}=\rho=r-d=n-m \text {, }
$$

where the operator $\Lambda_{0}^{*}$ is the adjoint one to $\Lambda_{0}$;

(2) the homogeneous boundary value problem (3.9) has $r$ and only $r$ linearly independent solutions $X_{r}(t) c_{r}$, for all $c_{r} \in \mathbb{R}^{r}\left(\operatorname{dim} \operatorname{ker} \Lambda_{0}=r=n-\operatorname{rank} Q=\right.$ $\left.n-n_{1}\right)$; 
(3) the nonhomogeneous boundary value problem (3.1) and (3.2) is solvable for those and only those $\varphi(t) \in L_{p}^{n}[a, b]$ and $\alpha \in \mathbb{R}^{m}$ which satisfy (3.5) $\left(\operatorname{dim} \operatorname{ker} \Lambda_{0}^{*}=d=m-\operatorname{rank} Q^{*}=m-n_{1}\right)$ and its solutions form the $r$ parametric family (3.8).

These results will essentially be applied for obtaining new existence conditions for the solutions of perturbed linear and nonlinear boundary value problems for delay equations.

Remark 3.2. If the vector functional $l$ satisfies the relation

$$
l \int_{a}^{b} K(\cdot, \tau) \varphi(\tau) d \tau=\int_{a}^{b} l K(\cdot, \tau) \varphi(\tau) d \tau
$$

then the generalized Green operator $(G \varphi)(t)$ obtains the form

$$
(G \varphi)(t)=\int_{a}^{b} G(t, \tau) \varphi(\tau) d \tau
$$

The $n \times n$ matrix $G(t, \tau)$ is the kernel of the integral representation of the operator $(G \varphi)(t)$ and has the form

$$
G(t, \tau)=K(t, \tau)-X(t) Q^{+} l K(\cdot, \tau)
$$

and is called generalized Green matrix. Without loss of generality, we will assume below that condition (3.13) is fulfilled.

For example, the relation (3.13) holds for periodic $l z:=z(a)-z(b)=0$ and for multipoint $l z=\sum_{i=1}^{k} M_{i} z\left(t_{i}\right)$ boundary conditions as well as for the conditions of the form of Riemann-Stieltjes integral

$$
l z=\int_{a}^{b} d \Phi(t) z(t)
$$

where $\Phi(t)$ is an $m \times n$ matrix whose components are functions with bounded variation on $[a, b]$. In the last case,

$$
l K(\cdot, \tau)=\int_{\tau}^{b} d \Phi(t) K(t, \tau)
$$

because $K(t, \tau) \equiv 0$ for $t<\tau$.

Remark 3.3. The solvability condition (3.5) for problem (3.1) and (3.2) holds provided that the initial function $\psi$ is appropriately chosen. In fact, using (2.3), we can represent condition (3.5) in the form

$$
P_{Q_{d}^{*}}\left\{\alpha-l \int_{a}^{b} K(\cdot, \tau)\left[g(\tau)+\sum_{i=1}^{k} A_{i}(\tau) \psi^{h_{i}}(\tau)\right] d \tau\right\}=0
$$


This allows us to get the solvability of problem (3.1) and (3.2) by varying the function $\psi$. But, if nonhomogeneities $g(t) \in L_{p}^{n}[a, b]$ and $\alpha \in \mathbb{R}^{m}$ and the initial vector function $\psi: \mathbb{R}^{1} \backslash[a, b] \rightarrow \mathbb{R}^{n}$ are arbitrary, then the solvability condition (3.5) for problem (3.1) and (3.2) does not hold. So, it is necessary to suggest a method for regularization of a boundary value problem which is not everywhere solvable.

\section{Perturbed boundary value problems}

Consider the perturbed nonhomogeneous linear boundary value problem (1.1) and (1.3), which, in view of (2.2) and (2.3), can be rewritten in the form

$$
\dot{z}(t)=A(t)\left(S_{h} z\right)(t)+\varepsilon B(t)\left(S_{h} z\right)(t)+\varphi(t), \quad l z=\alpha, \quad t \in[a, b] .
$$

As before, we will assume that $A(t)=\left(A_{1}(t), \ldots, A_{k}(t)\right)$ and $B(t)=\left(B_{1}(t), \ldots\right.$, $\left.B_{k}(t)\right)$ are $n \times N$ matrices $(N=n k)$ consisting, respectively, of $n \times n$ matrices $A_{i}(t) \in L_{p}^{n}[a, b]$ and $B_{i}(t) \in L_{p}^{n}[a, b]$. Assume that the generating boundary value problem

$$
\dot{z}(t)=A(t)\left(S_{h} z\right)(t)+\varphi(t), \quad l z=\alpha,
$$

which follows from (4.1) for $\varepsilon=0$, has no solution for arbitrary nonhomogeneities $\varphi(t) \in L_{p}^{n}[a, b]$ and $\alpha \in \mathbb{R}^{m}$. Then Theorem 3.1 shows that the solvability criterion (3.5) does not hold for problem (4.2) because the nonhomogeneities are arbitrary. Thus we arrive at the following question.

Question 4.1. Is it possible to make problem (4.2) solvable by means of linear perturbations and, if it is possible, then what kind should be the perturbations $B_{i}(t)$ and the delays $h_{i}(t)$ in order to make the boundary value problem (4.1) everywhere solvable?

We can answer this question with the help of the $d \times r$ matrix

$$
B_{0}=\int_{a}^{b} H(\tau) B(\tau)\left(S_{h} X_{r}\right)(\tau) d \tau, \quad H(\tau)=P_{Q_{d}^{*}} l K(\cdot, \tau),
$$

the construction of which involves the coefficients of problem (4.1). Using the method of [10] we can find conditions when solutions of the boundary value problem (4.1) appear in the form of Laurent series (in powers of a small parameter $\varepsilon$ ) with finite number terms of negative power of $\varepsilon$.

Below we will prove a statement, which enables us to solve the above problem. In order to state this result, we remind that by $P_{B_{0}}$ we denote an $r \times r$ matrixorthoprojector projecting $\mathbb{R}^{r}$ onto the null-space $N(B)$ of the $d \times r$ matrix $B_{0}$ 
and by $P_{B_{0}^{*}}$ we denote a $d \times d$ matrix-orthoprojector projecting $\mathbb{R}^{d}$ onto the nullspace $N\left(B_{0}^{*}\right)$ of the $r \times d$ matrix $B_{0}^{*}=B_{0}^{t}$. Now we can formulate the following lemma.

Lemma 4.2. Consider the boundary value problem (4.1) and assume that for arbitrary nonhomogeneities $\varphi(t) \in L_{p}^{n}[a, b]$ and $\alpha \in \mathbb{R}^{m}$ the generating boundary value problem (4.2) has no solutions.

If the equivalent relations

$$
P_{B_{0}^{*}}=0 \Longleftrightarrow \operatorname{rank} B_{0}=d
$$

hold, then for arbitrary $\varphi(t) \in L_{p}^{n}[a, b]$ and $\alpha \in \mathbb{R}^{m}$ the boundary value problem (4.1) has at least one solution in the form of the series

$$
z(t, \varepsilon)=\sum_{i=-1}^{\infty} \varepsilon^{i} z_{i}(t)
$$

converging for $\varepsilon \in\left(0, \varepsilon_{*}\right]$, where $\varepsilon_{*}$ is an appropriate constant characterizing the domain of the convergence of the series (4.5).

Proof. Substitute (4.5) into (4.1) and equate the coefficients at equal powers of $\varepsilon$. For $\varepsilon^{-1}$, we obtain the homogeneous boundary value problem

$$
\dot{z}_{-1}=A(t)\left(S_{h} z_{-1}\right)(t), \quad l z_{-1}=0,
$$

which determines $z_{-1}(t)$.

By the hypotheses of Theorem 3.1, the homogeneous boundary value problem (4.6) has an $r$-parametric $\left(r=n-n_{1}\right)$ family of solutions $z_{-1}\left(t, c_{-1}\right)=$ $X_{r}(t) \mathcal{c}_{-1}$, where the $r$-dimensional column vector $c_{-1} \in \mathbb{R}^{r}$ can be determined from the solvability condition of the problem for $z_{0}(t)$.

For $\varepsilon^{0}$, we get the boundary value problem

$$
\dot{z}_{0}=A(t) z_{0}+B(t)\left(S_{h} z_{-1}\right)(t)+\varphi(t), \quad l z_{0}=\alpha,
$$

which determines $z_{0}(t)$.

It is an implication of Theorem 3.1 that the solvability criterion for problem (4.7) has the form

$$
P_{Q_{d}^{*}} \alpha-\int_{a}^{b} H(\tau)\left\{\varphi(\tau)+B(\tau)\left(S_{h} X_{r}\right)(\tau) c_{-1}\right\} d \tau=0
$$

from which we receive with respect to $c_{-1} \in \mathbb{R}^{r}$ the algebraic system

$$
B_{0} c_{-1}=P_{Q_{d}^{*}} \alpha-\int_{a}^{b} H(\tau) \varphi(\tau) d \tau
$$


where

$$
B_{0}=\int_{a}^{b} H(\tau) B(\tau)\left(S_{h} X_{r}\right)(\tau) d \tau, \quad H(\tau)=P_{Q_{d}^{*}} l K(\cdot, \tau) .
$$

The last system is solvable for arbitrary $\varphi(t) \in L_{p}^{n}[a, b]$ and $\alpha \in \mathbb{R}^{m}$ if and only if the condition $P_{B_{0}^{*}}=0$ is satisfied. The system (4.9) becomes resolvable with respect to $c_{-1} \in \mathbb{R}^{r}$ up to an arbitrary constant vector $P_{B_{0}} c$ (for all $c \in \mathbb{R}^{r}$ ) from the null-space of matrix $B_{0}$ with

$$
c_{-1}=-B_{0}^{+}\left\{P_{Q_{d}^{*}} \alpha-\int_{a}^{b} H(\tau) \varphi(\tau) d \tau\right\}+P_{B_{0}} c .
$$

This solution can be rewritten in the form

$$
c_{-1}=\bar{c}_{-1}+P_{B_{\rho}} c_{\rho} \quad \forall c_{\rho} \in \mathbb{R}^{\rho},
$$

where

$$
\bar{c}_{-1}=-B_{0}^{+}\left\{P_{Q_{d}^{*}} \alpha-\int_{a}^{b} H(\tau) \varphi(\tau) d \tau\right\}
$$

and $P_{B_{\rho}}$ is an $(r \times \rho)$-dimensional matrix whose columns are complete set of $\rho$ linearly independent columns of $(r \times r)$-dimensional matrix $P_{B_{0}}$, with

$$
\rho=\operatorname{rank} P_{B_{0}}=r-\operatorname{rank} B_{0}=r-d=n-m .
$$

So, for the solutions of problem (4.6) we have the following expression:

$$
\begin{gathered}
z_{-1}\left(t, c_{\rho}\right)=\bar{z}_{-1}\left(\cdot, \bar{c}_{-1}\right)+X_{r}(t) P_{B_{\rho}} c_{\rho} \quad \forall c_{\rho} \in \mathbb{R}^{\rho}, \\
\bar{z}_{-1}\left(t, \bar{c}_{-1}\right)=X_{r}(t) \bar{c}_{-1} .
\end{gathered}
$$

Assuming that (4.4) holds, the boundary value problem (4.7) has the $r$-parametric family of solutions

$$
\begin{aligned}
z_{0}\left(t, c_{0}\right)= & X_{r}(t) c_{0}+X(t) Q^{+} \alpha \\
& +\int_{a}^{b} G(t, \tau)\left[\varphi(\tau)+B(\tau) S_{h}\left(\bar{z}_{-1}\left(\cdot, \bar{c}_{-1}\right)+X_{r}(\cdot) P_{B_{\rho}} c_{\rho}\right)(\tau)\right] d \tau
\end{aligned}
$$

Here $c_{0}$ is an $r$-dimensional constant vector, which is determined at the next step from the solvability condition of the boundary value problem for $z_{1}(t)$. 
For $\varepsilon^{1}$, we get the boundary value problem

$$
\dot{z}_{1}=A(t) z_{1}+B(t)\left(S_{h} z_{0}\right)(t), \quad l z_{1}=0,
$$

which determines $z_{1}(t)$. The solvability criterion for problem (4.17) has the form

$$
\begin{aligned}
& \int_{a}^{b} H(\tau) B(\tau) S_{h}\{ X_{r}(\cdot) c_{0}+X(\cdot) Q^{+} \alpha \\
&+\int_{a}^{b} G(\cdot, s)\left[\varphi(s)+B(s) S_{h}\right. \\
&\left.\left.\quad \times\left(\bar{z}_{-1}\left(\cdot, \bar{c}_{-1}\right)+X_{r}(\cdot) P_{B_{\rho}} c_{\rho}\right)(s)\right] d s\right\}(\tau) d \tau=0
\end{aligned}
$$

or equivalently the form

$$
\begin{aligned}
B_{0} c_{0}=\int_{a}^{b} H(\tau) B(\tau) S_{h}\{ & X(\cdot) Q^{+} \alpha \\
& +\int_{a}^{b} G(\cdot, s)\left[\varphi(s)+B(s) S_{h}\right. \\
& \left.\left.\quad \times\left(\bar{z}_{-1}\left(\cdot, \bar{c}_{-1}\right)+X_{r}(\cdot) P_{B_{\rho}} c_{\rho}\right)(s)\right] d s\right\}(\tau) d \tau .
\end{aligned}
$$

The algebraic system (4.19) has the following family of solutions:

$$
\begin{aligned}
c_{0}= & B_{0}^{+} \int_{a}^{b} H(\tau) B(\tau) S_{h} \\
& \times\left\{X(\cdot) Q^{+} \alpha+\int_{a}^{b} G(\cdot, s)\left[\varphi(s)+B(s)\left(S_{h} \bar{z}_{-1}\left(\cdot, \bar{c}_{-1}\right)\right)(s)\right] d s\right\}(\tau) d \tau \\
& +\left[I_{r}+B_{0}^{+} \int_{a}^{b} H(\tau) B(\tau) S_{h}\left\{\int_{a}^{b} G(\cdot, s) B(s)\left(S_{h} X_{r}(\cdot)\right)(s) d s\right\}(\tau) d \tau\right] P_{B_{\rho}} c_{\rho} \\
= & \bar{c}_{0}+[\cdot, \cdot, \cdot] P_{B_{\rho}} c_{\rho},
\end{aligned}
$$

where

$$
\begin{array}{rl}
\bar{c}_{0}=B_{0}^{+} \int_{a}^{b} & H(\tau) B(\tau) S_{h} \\
& \times\left\{X(\cdot) Q^{+} \alpha+\int_{a}^{b} G(\cdot, s)\left[\varphi(s)+B(s)\left(S_{h} \bar{z}_{-1}\left(\cdot, \bar{c}_{-1}\right)\right)(s)\right] d s\right\}(\tau) d \tau, \\
{[\cdot, \cdot, \cdot]} & =I_{r}+B_{0}^{+} \int_{a}^{b} H(\tau) B(\tau) S_{h}\left\{\int_{a}^{b} G(\cdot, s) B(s)\left(S_{h} X_{r}(\cdot)\right)(s) d s\right\}(\tau) d \tau .
\end{array}
$$


So, for the $\rho$-parametric family of solutions of problem (4.6) we have the following expression:

$$
z_{0}\left(t, c_{\rho}\right)=\bar{z}_{0}\left(t, \bar{c}_{0}\right)+\bar{X}_{0}(t) P_{B_{\rho}} c_{\rho} \quad \forall c_{\rho} \in \mathbb{R}^{\rho}
$$

where

$$
\begin{aligned}
\bar{z}_{0}\left(t, \bar{c}_{0}\right)= & X_{r}(t) \bar{c}_{0}+X(t) Q^{+} \alpha+\int_{a}^{b} G(t, \tau)\left[\varphi(\tau)+B(\tau)\left(S_{h} \bar{z}_{-1}\left(\cdot, \bar{c}_{-1}\right)\right)(\tau)\right] d \tau, \\
\bar{X}_{0}(t)= & X_{r}(t)\left[I_{r}+B_{0}^{+} \int_{a}^{b} H(\tau) B(\tau) S_{h}\left\{\int_{a}^{b} G(\cdot, s) B(s)\left(S_{h} X_{r}(\cdot)\right)(s) d s\right\}(\tau) d \tau\right] \\
& +\int_{a}^{b} G(t, \tau) B(\tau)\left(S_{h} X_{r}(\cdot)\right)(\tau) d \tau .
\end{aligned}
$$

Again, assuming that (4.4) holds, the boundary value problem (4.17) has the $r$-parametric family of solutions

$$
z_{1}\left(t, c_{1}\right)=X_{r}(t) c_{1}+\int_{a}^{b} G(t, \tau) B(\tau) S_{h}\left(\bar{z}_{0}\left(\cdot, \bar{c}_{0}\right)+\bar{X}_{0}(\cdot) P_{B_{\rho}} c_{\rho}\right)(\tau) d \tau
$$

Here $c_{1}$ is an $r$-dimensional constant vector, which is determined at the next step from the solvability condition of the boundary value problem for $z_{2}(t)$ :

$$
\dot{z}_{2}=A(t) z_{2}+B(t)\left(S_{h} z_{1}\right)(t), \quad l z_{2}=0 .
$$

The solvability criterion for problem (4.25) has the form

$$
\int_{a}^{b} H(\tau) B(\tau) S_{h}\left\{X_{r}(\cdot) c_{1}+\int_{a}^{b} G(\cdot, s) B(s) S_{h}\left(\bar{z}_{0}\left(\cdot, \bar{c}_{0}\right)+\bar{X}_{0}(\cdot) P_{B_{\rho}} c_{\rho}\right)(s) d s\right\}(\tau) d \tau=0
$$

or the form

$$
\begin{aligned}
& B_{0} c_{1} \\
& =-\int_{a}^{b} H(\tau) B(\tau)\left(S_{h}\left\{\int_{a}^{b} G(\cdot, s) B(s) S_{h}\left(\bar{z}_{0}\left(\cdot, \bar{c}_{0}\right)+\bar{X}_{0}(\cdot) P_{B_{\rho}} c_{\rho}\right)(s) d s\right\}\right)(\tau) d \tau .
\end{aligned}
$$

Under condition (4.4), the last equation has the $\rho$-parametric family of solutions

$$
c_{1}=\bar{c}_{1}+\{\cdot, \cdot, \cdot\}
$$


where

$$
\begin{aligned}
\bar{c}_{1} & =-B_{0}^{+} \int_{a}^{b} H(\tau) B(\tau)\left(S_{h}\left\{\int_{a}^{b} G(\cdot, s) B(s)\left(S_{h} \bar{z}_{0}\left(\cdot, \bar{c}_{0}\right)\right)(s) d s\right\}\right)(\tau) d \tau, \\
\{\cdot, \cdot, \cdot \cdot\} & \\
& =\left\{I_{r}-B_{0}^{+} \int_{a}^{b} H(\tau) B(\tau)\left(S_{h}\left\{\int_{a}^{b} G(\cdot, s) B(s)\left(S_{h} \bar{X}_{0}(\cdot)\right)(s) d s\right\}\right)(\tau) d \tau\right\} P_{B_{\rho}} c_{\rho} .
\end{aligned}
$$

So, for the coefficient $z_{1}\left(t, c_{1}\right)=z_{1}\left(t, c_{\rho}\right)$ we have the following expression:

$$
z_{1}(t, \rho)=\bar{z}_{1}\left(t, \bar{c}_{1}\right)+\bar{X}_{1}(t) P_{B_{\rho}} c_{\rho} \quad \forall c_{\rho} \in \mathbb{R}^{\rho},
$$

where

$$
\begin{aligned}
\bar{z}_{1}\left(t, \bar{c}_{1}\right)= & X_{r}(t) \bar{c}_{1}+\int_{a}^{b} G(t, \tau) B(\tau)\left(S_{h} \bar{z}_{0}\left(\cdot, \bar{c}_{0}\right)\right)(\tau) d \tau, \\
\bar{X}_{1}(t)= & X_{r}(t)\left[I_{r}-B_{0}^{+} \int_{a}^{b} H(\tau) B(\tau) S_{h}\left\{\int_{a}^{b} G(\cdot, s) B(s)\left(S_{h} \bar{X}_{0}(\cdot)\right)(s) d s\right\}(\tau) d \tau\right] \\
& +\int_{a}^{b} G(t, \tau) B(\tau)\left(S_{h} \bar{X}_{0}(\cdot)\right)(\tau) d \tau .
\end{aligned}
$$

Continuing this process, assuming that (4.4) holds, it follows by induction that the coefficients $z_{i}\left(t, c_{i}\right)=z_{i}\left(t, c_{\rho}\right)$ of the series (4.5) can be determined from the relevant boundary value problems as follows:

$$
z_{i}(t, \rho)=\bar{z}_{i}\left(t, \bar{c}_{i}\right)+\bar{X}_{i}(t) P_{B_{\rho}} c_{\rho} \quad \forall c_{\rho} \in \mathbb{R}^{\rho},
$$

where

$$
\begin{aligned}
\bar{z}_{i}\left(t, \bar{c}_{i}\right)= & X_{r}(t) \bar{c}_{1}+\int_{a}^{b} G(t, \tau) B(\tau) S_{h} \bar{z}_{i-1}\left(\cdot, \bar{c}_{i-1}\right)(\tau) d \tau \\
\bar{c}_{i}= & -B^{+} \int_{a}^{b} H(\tau) B(\tau)\left(S_{h}\left\{\int_{a}^{b} G(\cdot, s) B(s) S_{h} \bar{z}_{i-1}\left(\cdot, \bar{c}_{i-1}\right)(s) d s\right\}\right)(\tau) d \tau \\
i=1,2, \ldots, & \\
\bar{X}_{i}(t)= & X_{r}(t)\left[I_{r}+B_{0}^{+} \int_{a}^{b} H(\tau) B(\tau) S_{h}\left\{\int_{a}^{b} G(\cdot, s) B(s)\left(S_{h} \bar{X}_{i-1}(\cdot)\right)(s) d s\right\}(\tau) d \tau\right] \\
& +\int_{a}^{b} G(t, \tau) B(\tau)\left(S_{h} \bar{X}_{i-1}(\cdot)\right)(\tau) d \tau, \quad i=0,1,2, \ldots, \bar{X}_{-1}(t)=X_{r}(t) .
\end{aligned}
$$

Since the convergence of the series (4.5) can be proved by traditional methods of majorization, the proof of the lemma is complete. 
From the above lemma we have the following conclusions.

The boundary value problem (4.1) determines the bounded operator

$$
\left(\Lambda_{\varepsilon} z\right)(t) \stackrel{\text { def }}{=} \operatorname{col}\left[\dot{z}(t)-A(t)\left(S_{h} z\right)(t)-\varepsilon B(t)\left(S_{h} z\right)(t), l z\right]
$$

which is acting from the space $D_{p}^{n}[a, b]$ to the space $L_{p}^{n}[a, b] \times \mathbb{R}^{m}, 1<p<\infty$. Under the assumption (4.4), problem (4.1) is always solvable in the Banach spaces under consideration. This means that the image of the operator $\Lambda_{\varepsilon}$ coincides with the whole space $L_{p}^{n}[a, b] \times \mathbb{R}^{m}$, that is, $\operatorname{Im} \Lambda_{\varepsilon}=L_{p}^{n}[a, b] \times \mathbb{R}^{m}$. Therefore, $\Lambda_{\varepsilon}$ is a normally solvable operator (see $[5,7]$ ), while the boundary value problem adjoint to the homogeneous one

$$
\dot{z}(t)=A(t)\left(S_{h} z\right)(t)+\varepsilon B(t)\left(S_{h} z\right)(t), \quad l z=0 \in \mathbb{R}^{m},
$$

has only trivial solutions, that is, $\operatorname{dim} \operatorname{ker} \Lambda_{\varepsilon}^{*}=0, \varepsilon \neq 0$, where the operator $\Lambda_{\varepsilon}^{*}$ is the adjoint one to $\Lambda_{\varepsilon}$ in the corresponding spaces. Note that our problem does not need the construction of the adjoint problem. Such a construction for the unperturbed boundary value problem (3.1) and (3.2) is given in [1, page 36].

As it is shown in the proof of Lemma 4.2, $\operatorname{dim} \operatorname{ker} \Lambda_{\varepsilon}=\rho=r-d$. This, together with the above-mentioned property $\operatorname{dim} \operatorname{ker} \Lambda_{\varepsilon}^{*}=0$, means that the normally solvable operator $\Lambda_{\varepsilon}$ is a Fredholm one. Now, it is not difficult to see that for the differential operator (4.34) with delayed arguments, the well-known fact from the theory of operators (see [5] or [7, page 86]), concerning the maintenance under small perturbations of the index of the Fredholm operator $\Lambda_{0}$ (3.11), is satisfied. Indeed, since by Theorem 3.1,

$$
\operatorname{dim} \operatorname{ker} \Lambda_{0}=r, \quad \operatorname{dim} \operatorname{ker} \Lambda_{0}^{*}=d,
$$

and by Lemma 4.2,

$$
\operatorname{dim} \operatorname{ker} \Lambda_{\varepsilon}=r-d, \quad \operatorname{dim} \operatorname{ker} \Lambda_{\varepsilon}^{*}=0, \quad \varepsilon \neq 0,
$$

it follows that

$$
\text { ind } \Lambda_{0}=\operatorname{ind} \Lambda_{\varepsilon}
$$

From the previous discussion we have the following theorem.

Theorem 4.3. Consider the boundary value problem

$$
\dot{z}(t)=A(t)\left(S_{h} z\right)(t)+\varepsilon B(t)\left(S_{h} z\right)(t)+\varphi(t), \quad l z=\alpha \in \mathbb{R}^{m},
$$


and assume that for arbitrary nonhomogeneities $\varphi(t) \in L_{p}^{n}[a, b]$ and $\alpha \in \mathbb{R}^{m}$ the generating boundary value problem (4.2) has no solutions.

If the condition

$$
\operatorname{rank}\left[B_{0}=\int_{a}^{b} H(\tau) B(\tau)\left(S_{h} X_{r}\right)(\tau) d \tau\right]=d=m-n_{1} \quad\left(\operatorname{rank} Q=n_{1}\right)
$$

holds, then

(1) the operator $\Lambda_{\varepsilon}: D_{p}^{n}[a, b] \rightarrow L_{p}^{n}[a, b] \times \mathbb{R}^{m}(1<p<\infty)$ defined by (4.34) is a Fredholm one with

$$
\begin{aligned}
& \operatorname{ind} \Lambda_{\varepsilon}=\operatorname{dim} \operatorname{ker} \Lambda_{\varepsilon}-\operatorname{dim} \operatorname{ker} \Lambda_{\varepsilon}^{*}=\rho=r-d=n-m, \\
& \operatorname{ind} \Lambda_{0}=\operatorname{dim} \operatorname{ker} \Lambda_{0}-\operatorname{dim} \operatorname{ker} \Lambda_{0}^{*}=\rho=r-d=n-m,
\end{aligned}
$$

where the operator $\Lambda_{\varepsilon}^{*}$ is the adjoint one to $\Lambda_{\varepsilon},\left(\operatorname{dim} \operatorname{ker} \Lambda_{0}=r, \operatorname{dim} \operatorname{ker} \Lambda_{0}^{*}\right.$ $=d$ );

(2) the homogeneous boundary value problem (4.35) has a $\rho$-parametric family of solutions

$$
z_{0}\left(t, \varepsilon, c_{\rho}\right)=\sum_{i=-1}^{\infty} \varepsilon^{i} \bar{X}_{i}(t) P_{B_{\rho}} c_{\rho} \quad \forall c_{\rho} \in \mathbb{R}^{\rho},\left(\rho=\operatorname{dim} \operatorname{ker} \Lambda_{\varepsilon}\right)
$$

with the properties

$$
z_{0}\left(\cdot, \mathcal{\varepsilon}, c_{\rho}\right) \in D_{p}^{n}[a, b], \quad \dot{z}_{0}\left(\cdot, \mathcal{\varepsilon}, c_{\rho}\right) \in L_{p}^{n}[a, b], \quad z_{0}\left(t, \cdot, c_{\rho}\right) \in C\left(0, \varepsilon_{*}\right]
$$

(3) the boundary value problem adjoint to (4.35) has only trivial solutions $\left(\operatorname{dim} \operatorname{ker} \Lambda_{\varepsilon}^{*}=0, \varepsilon \neq 0\right)$;

(4) for arbitrary $\varphi(t) \in L_{p}^{n}[a, b]$ and $\alpha \in \mathbb{R}^{m}$ the boundary value problem (4.39) has a $\rho$-parametric set of solutions $z(t, \varepsilon)=z\left(t, \varepsilon, c_{\rho}\right)$ with the properties

$$
z\left(\cdot, \varepsilon, c_{\rho}\right) \in D_{p}^{n}[a, b], \quad \dot{z}\left(\cdot, \varepsilon, c_{\rho}\right) \in L_{p}^{n}[a, b], \quad z\left(t, \cdot, c_{\rho}\right) \in C\left(0, \varepsilon_{*}\right],
$$

in the form of the series

$$
z\left(t, \varepsilon, c_{\rho}\right)=\sum_{i=-1}^{\infty} \varepsilon^{i}\left[\bar{z}_{i}\left(t, \bar{c}_{i}\right)+\bar{X}_{i}(t) P_{B_{\rho}} c_{\rho}\right] \quad \forall c_{\rho} \in \mathbb{R}^{\rho},
$$

converging for $\mathcal{\varepsilon} \in\left(0, \varepsilon_{*}\right]$, where $\varepsilon_{*}$ is as in Lemma 4.2 and the coefficients $\bar{z}_{i}\left(t, \bar{c}_{i}\right), \bar{c}_{i}$, and $\bar{X}_{i}(t)$ can be determined from (4.32). 
In the case when the number $m$ of boundary conditions is equal to the dimension $n$ of the differential system (4.39), from the condition (4.40) we have

$$
\operatorname{rank}\left[B_{0}=\int_{a}^{b} H(\tau) B(\tau)\left(S_{h} X_{r}\right)(\tau) d \tau\right]=r=d
$$

and from Theorem 4.3 we have the following corollary.

Corollary 4.4. Consider the boundary value problem

$$
\dot{z}(t)=A(t)\left(S_{h} z\right)(t)+\varepsilon B(t)\left(S_{h} z\right)(t)+\varphi(t), \quad l z=\alpha \in \mathbb{R}^{n},
$$

and assume that for arbitrary nonhomogeneities $\varphi(t) \in L_{p}^{n}[a, b]$ and $\alpha \in \mathbb{R}^{n}$ the generating boundary value problem (4.2) has no solutions. If the condition

$$
\operatorname{det} B_{0} \neq 0
$$

holds, then

(1) the operator $\Lambda_{\varepsilon}: D_{p}^{n}[a, b] \rightarrow L_{p}^{n}[a, b] \times \mathbb{R}^{n}$ defined by

$$
\left(\Lambda_{\varepsilon} z\right)(t) \stackrel{\text { def }}{=} \operatorname{col}\left[\dot{z}(t)-A(t)\left(S_{h} z\right)(t)-\varepsilon B(t)\left(S_{h} z\right)(t), l z\right]
$$

is a Fredholm index zero operator with

$$
\text { ind } \Lambda_{\varepsilon}=\operatorname{dim} \operatorname{ker} \Lambda_{\varepsilon}-\operatorname{dim} \operatorname{ker} \Lambda_{\varepsilon}^{*}=0,
$$

$\operatorname{ind} \Lambda_{0}=\operatorname{dim} \operatorname{ker} \Lambda_{0}-\operatorname{dim} \operatorname{ker} \Lambda_{0}^{*}=0 \quad\left(\operatorname{dim} \operatorname{ker} \Lambda_{0}=\operatorname{dim} \operatorname{ker} \Lambda_{0}^{*}=r=d\right)$;

(2) the homogeneous boundary value problem

$$
\dot{z}(t)=A(t)\left(S_{h} z\right)(t)+\varepsilon B(t)\left(S_{h} z\right)(t), \quad l z=0 \in \mathbb{R}^{n}
$$

has only trivial solutions ( $\left.\operatorname{dim} \operatorname{ker} \Lambda_{\varepsilon}=0, \varepsilon \neq 0\right)$;

(3) the boundary value problem adjoint to (4.51) has only trivial solutions $\left(\operatorname{dim} \operatorname{ker} \Lambda_{\varepsilon}^{*}=0, \varepsilon \neq 0\right)$;

(4) for arbitrary $\varphi(t) \in L_{p}^{n}[a, b]$ and $\alpha \in \mathbb{R}^{n}$ the boundary value problem (4.47) has the unique solution $z(t, \varepsilon)$ with the properties

$z(\cdot, \varepsilon) \in D_{p}^{n}[a, b], \quad \dot{z}(\cdot, \varepsilon) \in L_{p}^{n}[a, b], \quad z(t, \cdot) \in C\left(0, \varepsilon_{*}\right]$,

in the form of the series

$$
z(t, \varepsilon)=\sum_{i=-1}^{\infty} \varepsilon^{i} \bar{z}_{i}\left(t, \bar{c}_{i}\right)
$$


converging for $\varepsilon \in\left(0, \varepsilon_{*}\right]$, where $\varepsilon_{*}$ is as in Lemma 4.2 and the coefficients $\bar{z}_{i}\left(t, \bar{c}_{i}\right), \bar{c}_{i}$ can be determined from (4.32).

Remark 4.5. If (4.40) does not hold, then in order to obtain sufficient conditions for existence of solutions of the boundary value problem (4.39) for arbitrary nonhomogeneities $\varphi(t) \in L_{p}^{n}[a, b]$ and $\alpha \in \mathbb{R}^{m}$, the solution $z(t, \varepsilon)$ of problem (4.39) is constructed in the form of series (4.5) with $i \leq-2$.

Remark 4.6. If

$$
\operatorname{rank}\left[B_{0}=\int_{a}^{b} H(\tau) B(\tau)\left(S_{h} X_{r}\right)(\tau) d \tau\right]=d,
$$

then the nonlinear boundary value problem with the measurable delays $h_{i}(t)$

$$
\begin{gathered}
\dot{z}(t)=\sum_{i=1}^{k} A_{i}(t) z\left(h_{i}(t)\right)+g(t)+\varepsilon \sum_{i=1}^{k} B_{i}(t) z\left(h_{i}(t)\right)+\varepsilon \sum_{i=1}^{k} R_{i}\left(z\left(h_{i}(t)\right), t, \varepsilon\right), \\
z(s)=\psi(s), \quad \text { if } s<a, \quad l z=\alpha \in \mathbb{R}^{m}, \quad t \in[a, b]
\end{gathered}
$$

has at least one solution $z(t, \varepsilon)$ with the properties

$$
z(\cdot, \varepsilon) \in D_{p}^{n}[a, b], \quad \dot{z}(\cdot, \varepsilon) \in L_{p}^{n}[a, b]
$$

where

$$
A_{i}(t), B_{i}(t), g(t), R_{i}(z, \cdot, \varepsilon) \in L_{p}^{n}[a, b], \quad R_{i}(z, t, \varepsilon)=o\left(z^{2}\right) .
$$

\section{Applications}

Example 5.1. Consider the linear boundary value problem for the delay differential equation

$$
\begin{gathered}
\dot{z}(t)=\varepsilon \sum_{i=1}^{k} B_{i}(t) z\left(h_{i}(t)\right)+g(t), \quad t \in[0, T], \\
z(s)=\psi(s), \quad \text { if } s<0, \quad z(0)=z(T),
\end{gathered}
$$

where $B_{i}(t)$ are $n \times n$ matrices, $B_{i}(t), g(t) \in L_{p}^{n}[0, T]$, and $\psi: \mathbb{R}^{1} \backslash[a, b] \rightarrow \mathbb{R}^{n}$, $h_{i}(t)$ are measurable functions. Using the symbols $S_{h_{i}}$ and $\psi^{h_{i}}$ (see (2.2), (2.3)), we arrive at the following operator system:

$$
\dot{z}(t)=\varepsilon B(t)\left(S_{h} z\right)(t)+\varphi(t), \quad l z=z(0)-z(T)=0,
$$


where $B(t)=\left(B_{1}(t), \ldots, B_{k}(t)\right)$ is an $n \times N$ matrix $(N=n k)$, and

$$
\varphi(t)=g(t)+\sum_{i=1}^{k} B_{i}(t) \psi^{h_{i}}(t) \in L_{p}^{n}[0, T] .
$$

It is easily verified that

$$
\begin{gathered}
X(t)=E, \quad \dot{z}(t)=0, \quad l X(\cdot)=Q=0, \quad P_{Q}=P_{Q^{*}}=E, \quad(r=n, d=m=n), \\
K(t, \tau)= \begin{cases}E, & 0 \leq \tau \leq t \leq T, \\
0, & \tau>t,\end{cases} \\
l K(\cdot, \tau)=K(0, \tau)-K(T, \tau)=-E, \\
H(\tau)=P_{Q^{*}} l K(\cdot, \tau)=-E .
\end{gathered}
$$

According to the representation (2.9), we have the following expressions:

$$
\begin{aligned}
\left(S_{h_{i}} E\right)(t) & =\chi_{h_{i}}(t, 0) E=E \begin{cases}1, & \text { if } 0 \leq h_{i}(t) \leq T \\
0, & \text { if } h_{i}(t)<0\end{cases} \\
B_{0} & =-\int_{0}^{T} H(\tau) B(\tau)\left(S_{h} E\right)(\tau) d \tau=-\int_{0}^{T} \sum_{i=1}^{k} B_{i}(\tau)\left(S_{h_{i}} E\right)(\tau) d \tau \\
& =-\sum_{i=1}^{k} \int_{0}^{T} B_{i}(\tau) \chi_{h_{i}}(\tau, 0) d \tau
\end{aligned}
$$

where $B_{0}$ is an $n \times n$ matrix.

If $\operatorname{det} B_{0} \neq 0$, then problem (5.1) has the unique solution $z(t, \varepsilon)$ with the properties

$$
z(\cdot, \varepsilon) \in D_{p}^{n}[0, T], \quad \dot{z}(\cdot, \varepsilon) \in L_{p}^{n}[0, T], \quad z(t, \cdot) \in C\left(0, \varepsilon_{*}\right]
$$

for arbitrary $g(t) \in L_{p}^{n}[0, T], \psi: \mathbb{R}^{1} \backslash[a, b] \rightarrow \mathbb{R}^{n}$, and for measurable delays $h_{i}(t)$. If, for example, $h_{i}(t)=t-\Delta_{i}$, where $0<\Delta_{i}=$ const $<T, i=1, \ldots, k$, then

$$
\begin{aligned}
\chi_{h_{i}}(t, 0) & = \begin{cases}1, & \text { if } 0 \leq h_{i}(t)=t-\Delta_{i} \leq T, \\
0, & \text { if } h_{i}(t)=t-\Delta_{i}<0,\end{cases} \\
& = \begin{cases}1, & \text { if } \Delta_{i} \leq t \leq T+\Delta_{i}, \\
0, & \text { if } t<\Delta_{i} .\end{cases}
\end{aligned}
$$


For this reason the $n \times n$ matrix $B_{0}$ can be rewritten in the form

$$
\begin{aligned}
B_{0} & =-\int_{0}^{T} H(\tau) \sum_{i=1}^{k} B_{i}(\tau) \chi_{h_{i}}(\tau, 0) d \tau \\
& =-\sum_{i=1}^{k} \int_{0}^{T} B_{i}(\tau) \chi_{h_{i}}(\tau, 0) d \tau \\
& =-\sum_{i=1}^{k} \int_{\Delta_{i}}^{T} B_{i}(\tau) d \tau,
\end{aligned}
$$

while the solvability condition of the boundary value problem (5.1) has the form

$$
\operatorname{det}\left[B_{0}=-\sum_{i=1}^{k} \int_{\Delta_{i}}^{T} B_{i}(\tau) d \tau\right] \neq 0 .
$$

In the case where there is no delay effect $\left(\Delta_{i}=0, i=1, \ldots, k\right)$, the last solvability condition coincides with such one of $[2,6]$.

Example 5.2. Consider the linear boundary value problem for the delay differential equation

$$
\begin{gathered}
\dot{z}(t)=\varepsilon \sum_{i=1}^{k} B_{i}(t) z\left(h_{i}(t)\right)+g(t), \quad t \in[0, T], \\
z(s)=\psi(s), \quad \text { if } s<0, \\
l z:=[1, \underbrace{0 \cdots 0}_{(n-1) \text { time }}] z(0)-[1, \underbrace{0 \cdots 0}_{(n-1) \text { time }}] z(T)=\alpha \in \mathbb{R} \quad(m=1) .
\end{gathered}
$$

Using the symbols $S_{h_{i}}$ and $\psi^{h_{i}}$, we arrive at the following boundary value problem for operator system:

$$
\begin{gathered}
\dot{z}(t)=\varepsilon B(t)\left(S_{h} z\right)(t)+\varphi(t), \\
l z:=[1, \underbrace{0 \cdots 0}_{(n-1) \text { time }}] z(0)-[1, \underbrace{0 \cdots 0}_{(n-1) \text { time }}] z(T)=\alpha \in \mathbb{R} \quad(m=1),
\end{gathered}
$$

where $B(t)=\left(B_{1}(t), \ldots, B_{k}(t)\right)$ is an $n \times N$ matrix $(N=n k)$, and

$$
\varphi(t)=g(t)+\sum_{i=1}^{k} B_{i}(t) \psi^{h_{i}}(t) \in L_{p}^{n}[0, T] .
$$


It is easy to see that

$$
\begin{gathered}
X(t)=E, \quad \dot{z}(t)=0, \quad l X(\cdot)=Q=[\underbrace{0 \cdots 0}_{n \text { time }}], \quad P_{Q}=E, \quad P_{Q^{*}}=1, \\
\left(\operatorname{rank} Q=n_{1}=0, r=n, d=m-n_{1}=1\right), \\
K(t, \tau)= \begin{cases}E, & 0 \leq \tau \leq t \leq T, \\
0, & \tau>t,\end{cases} \\
l K(\cdot, \tau)=\left[\begin{array}{c}
1, \underbrace{0 \cdots 0}_{(n-1) \text { time }}] K(0, \tau)-[1, \underbrace{0 \cdots 0}_{(n-1) \text { time }}] K(T, \tau)=-[1, \underbrace{0 \cdots 0}_{(n-1) \text { time }}], \\
H(\tau)=P_{Q^{*}} l K(\cdot, \tau)=-[1, \underbrace{0 \cdots 0}_{(n-1) \text { time }}] .
\end{array}\right.
\end{gathered}
$$

According to the representation (2.9), we have the following expression:

$$
\left(S_{h_{i}} E\right)(t)=\chi_{h_{i}}(t, 0) E=E \begin{cases}1, & \text { if } 0 \leq h_{i}(t) \leq T \\ 0, & \text { if } h_{i}(t)<0\end{cases}
$$

In order to obtain the solvability conditions for problem (5.10), it suffices to consider only the first row of the matrices

$$
B_{i}(t)=\left(\begin{array}{cccccc}
b_{11}^{(i)}(t) & b_{12}^{(i)}(t) & * & * & * & b_{1 n}^{(i)}(t) \\
* & * & * & * & * & * \\
* & * & * & * & * & *
\end{array}\right), \quad(i=1, \ldots, k) .
$$

Indeed, the $1 \times n$ matrix has the form

$$
\begin{aligned}
B_{0}=- & \int_{0}^{T} H(\tau) B(\tau)\left(S_{h} E\right)(\tau) d \tau \\
= & -\int_{0}^{T} H(\tau) \sum_{i=1}^{k} B_{i}(\tau)\left(S_{h_{i}} E\right)(\tau) d \tau \\
= & -\int_{0}^{T} H(\tau) \sum_{i=1}^{k} B_{i}(\tau) \chi_{h_{i}}(\tau, 0) d \tau \\
=- & {\left[\sum_{i=1}^{k} \int_{0}^{T} b_{11}^{(i)}(\tau) \chi_{h_{i}}(\tau, 0) d \tau, \sum_{i=1}^{k} \int_{0}^{T} b_{12}^{(i)}(\tau) \chi_{h_{i}}(\tau, 0) d \tau, \ldots\right.} \\
& \left.\sum_{i=1}^{k} \int_{0}^{T} b_{1 n}^{(i)}(\tau) \chi_{h_{i}}(\tau, 0) d \tau\right]
\end{aligned}
$$


If one of the inequalities

$$
\sum_{i=1}^{k} \int_{0}^{T} b_{1 j}^{(i)}(\tau) \chi_{h_{i}}(\tau, 0) d \tau \neq 0 \quad(j=1, \ldots, n)
$$

is true, then $\operatorname{rank} B_{0}=d=1$, and for arbitrary $\varphi(t) \in L_{p}^{n}[a, b], \alpha \in \mathbb{R}$, and for measurable delays $h_{i}(t)$ the boundary value problem (5.10) has a $\rho$-parametric set (where $\rho=n-1$ ) of solutions $z\left(t, c_{\rho}, \varepsilon\right)$ with the properties

$$
z\left(\cdot, c_{\rho}, \varepsilon\right) \in D_{p}^{n}[0, T], \quad \dot{z}\left(\cdot, c_{\rho}, \varepsilon\right) \in L_{p}^{n}[0, T], \quad z\left(t, c_{\rho}, \cdot\right) \in C\left(0, \varepsilon_{*}\right],
$$

in the form of the series (4.45), where $\varepsilon_{*}$ is as in Lemma 4.2 .

If, for example, $h_{i}(t)=t-\Delta_{i}$, where $0<\Delta_{i}=$ const $<T, i=1, \ldots, k$, then

$$
\chi_{h_{i}}(t, 0)= \begin{cases}1, & \text { if } \Delta_{i} \leq t \leq T+\Delta_{i} \\ 0, & \text { if } t<\Delta_{i}\end{cases}
$$

For this reason the $(1 \times n)$-dimensional matrix $B_{0}$ can be rewritten in the form

$$
\begin{aligned}
B_{0} & =-\int_{0}^{T} H(\tau) \sum_{i=1}^{k} B_{i}(\tau) \chi_{h_{i}}(\tau, 0) d \tau \\
& =-\left[\sum_{i=1}^{k} \int_{\Delta_{i}}^{T} b_{11}^{(i)}(\tau) d \tau, \sum_{i=1}^{k} \int_{\Delta_{i}}^{T} b_{12}^{(i)}(\tau) d \tau, \ldots, \sum_{i=1}^{k} \int_{\Delta_{i}}^{T} b_{1 n}^{(i)}(\tau) d \tau\right]
\end{aligned}
$$

and one of the solvability conditions of problem (5.10) is of the form

$$
\sum_{i=1}^{k} \int_{\Delta_{i}}^{T} b_{1 j}^{(i)}(\tau) d \tau \neq 0 \quad(j=1, \ldots, n) .
$$

\section{Acknowledgments}

The authors would like to thank the referee for making several helpful suggestions for improving the presentation of this paper. The essential part of the present work was finished while the first author was visiting the University of Ioannina during the winter of 2003. He would like to thank the Ministry of Economy and Finance of Hellenic Republik for providing the NATO Science Fellowship (Ref. No. DOO 850) and University of Ioannina for its hospitality. 


\section{References}

[1] N. V. Azbelev, V. P. Maksimov, and L. F. Rakhmatullina, Introduction to the Theory of Functional-Differential Equations, Nauka, Moscow, 1991 (Russian).

[2] A. A. Bŏ̌chuk, V. F. Zhuravlëv, and A. M. Samoĭlenko, Generalized Inverse Operators and Noether Boundary Value Problems, Proceedings of the Institute of Mathematics of the National Academy of Sciences of Ukraine, vol. 13, Natsīonal'na Akademīya Nauk Ukraïni Īnstitut Matematiki, Kiev, 1995 (Russian).

[3] J. K. Hale, Theory of Functional Differential Equations, Applied Mathematical Sciences, vol. 3, Springer-Verlag, New York, 1977.

[4] P. R. Halmos, Measure Theory, D. Van Nostrand, New York, 1950.

[5] T. Kato, Perturbation Theory for Linear Operators, Die Grundlehren der Mathematischen Wissenschaften, vol. 132, Springer-Verlag, New York, 1966.

[6] I. T. Kiguradze, Boundary value problems for systems of ordinary differential equations, Current Problems in Mathematics. Newest Results, Vol. 30 (Russian), Itogi Nauki i Tekhniki, Akad. Nauk SSSR Vsesoyuz. Inst. Nauchn. i Tekhn. Inform., Moscow, 1987, pp. 3-103 (Russian).

[7] S. G. Kreĭn, Linear Equations in a Banach Space, Izdat. Nauka, Moscow, 1971 (Russian).

[8] A. D. Myshkis, Linear Differential Equations with Retarded Argument, Izdat. Nauka, Moscow, 1972 (Russian).

[9] C. R. Rao and S. K. Mitra, Generalized Inverse of Matrices and Its Applications, John Wiley \& Sons, New York, 1971.

[10] M. I. Vishik and L. A. Lyusternik, Solvability of some problems concerning perturbations in case of matrices and selfadjoint and non-selfadjoint differential equations, Uspekhi Mat. Nauk 15 (1960), no. 3, 3-80 (Russian).

Alexander A. Boichuk: Institute of Mathematics, The National Academy of Science of Ukraine, 3 Tereshchenkivs'ka Street, 01601 Kyiv, Ukraine

Current address: Department of Applied Mathematics, Faculty of Natural Science, University of Zilina, J. M. Hurbana 15, 01026 Zilina, Slovak Republic

E-mail address: boichuk@imath.kiev.ua

Myron K. Grammatikopoulos: Department of Mathematics, University of Ioannina, 451

10 Ioannina, Hellas, Greece

E-mail address: mgrammat@cc.uoi.gr 


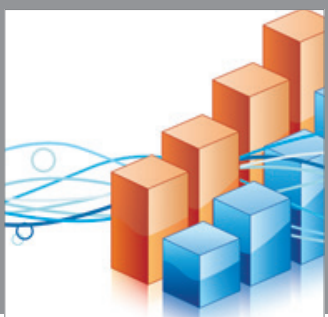

Advances in

Operations Research

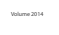

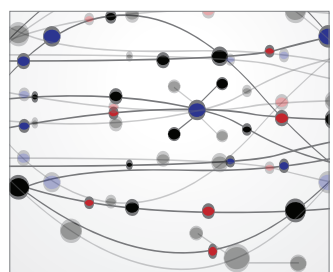

\section{The Scientific} World Journal
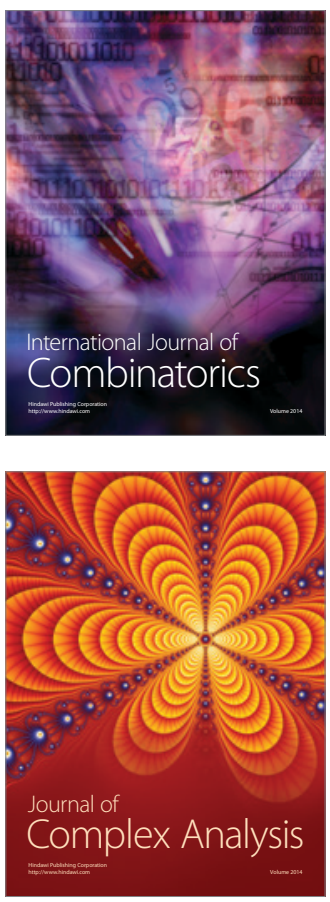

International Journal of

Mathematics and

Mathematical

Sciences
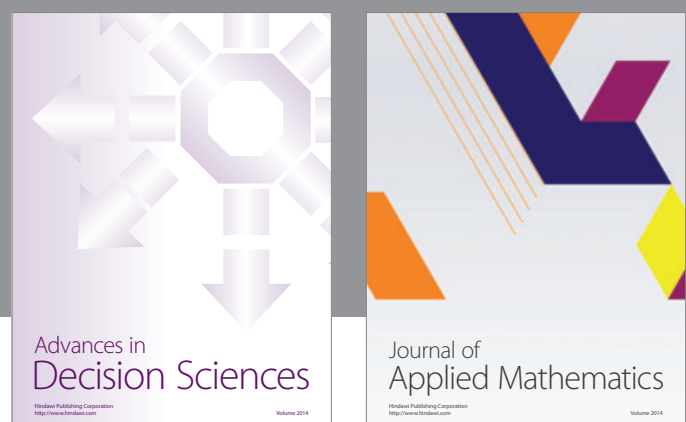

Journal of

Applied Mathematics
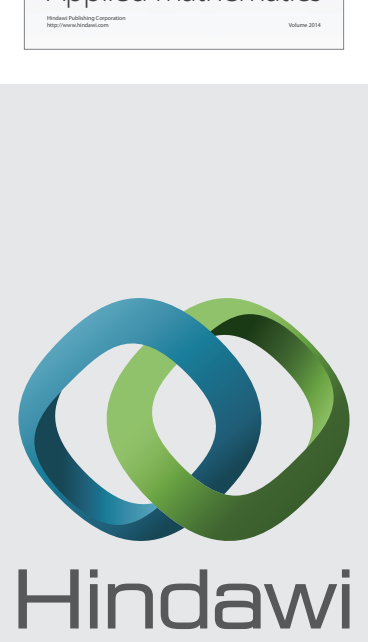

Submit your manuscripts at http://www.hindawi.com
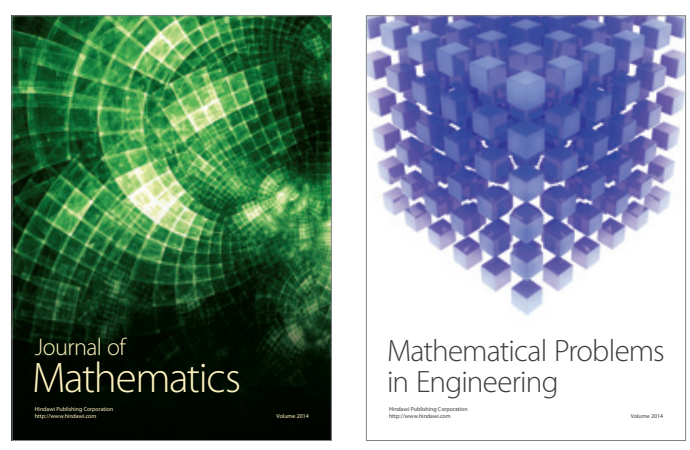

Mathematical Problems in Engineering
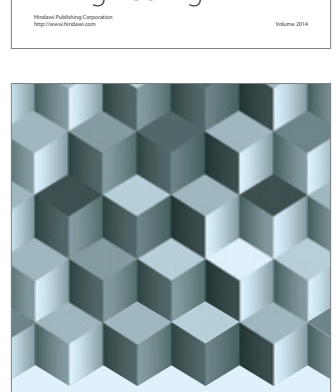

Journal of

Function Spaces
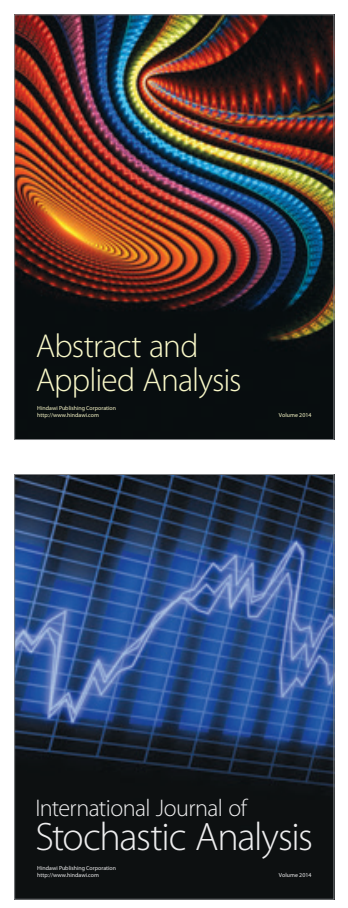

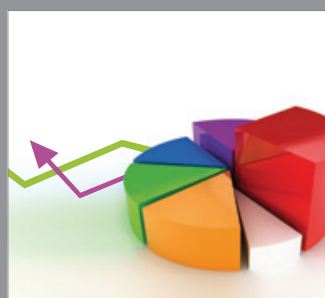

ournal of

Probability and Statistics

Promensencen
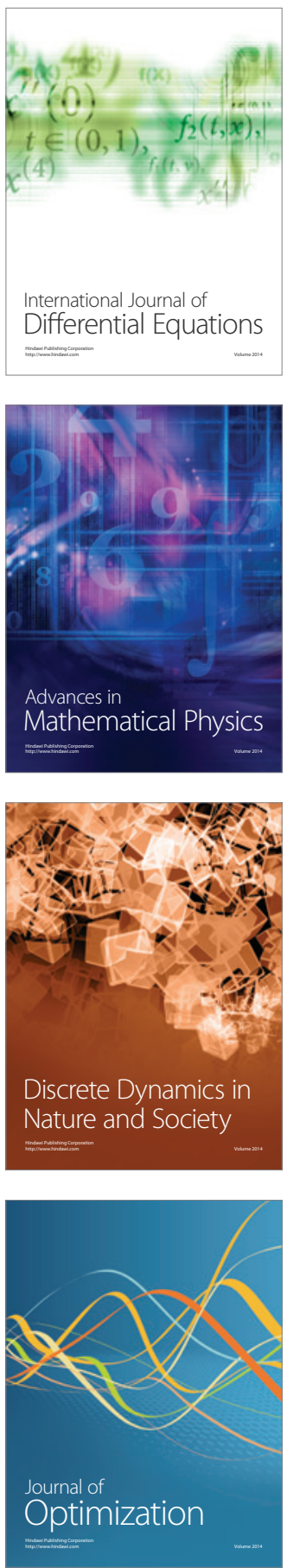